\title{
Comparison of Acceleration Plethysmogram Acquired Using Optical Signal and Pressure Signal
}

\author{
Revati Shriram ${ }^{1,3}$, Betty Martin ${ }^{2}$, M. Sundhararajan ${ }^{4}$ and Nivedita Daimiwal ${ }^{3}$ \\ ${ }^{1}$ Research Scholar, ECE Dept, Sathyabama University, Chennai, Tamil Nadu, \\ India \\ ${ }^{2}$ Sathyabama University, Chennai, Tamil Nadu, India \\ ${ }^{3}$ Cummins College of Engineering for Women, Pune, Maharashtra, India \\ ${ }^{4}$ Bharath University, Chennai, Tamil Nadu, India \\ revatishriram@yahoo.com
}

\begin{abstract}
Photoplethysmography (PPG) is an optical non-invasive technique to capture peripheral pulse, which changes with change in cardiac output with every heartbeat. Diagnostic information about cardiovascular condition can be obtained from this pulse. Reflection type of PPG sensor and Power Lab based piezoelectric pressure sensor is used to record the optical and mechanical pulse at various sites. PPG sensor is used to determine the properties of blood vessels and blood circulation dynamics such as crest time and delta $T$ from first derivative of the pulse and various ratios related to second derivative of the pulse. These obtained values are compared with the properties obtained from mechanical signal - Pressure Pulse (PP). Work was carried out by the authors to check the feasibility of using PP for the predicting the health/aging of blood vessels in near future. Statistical analysis of derivatives of PPG \& PP was carried out. Our results showed that most of the ratios obtained from PPG are same as that of the one obtained from PP. Effect of measurement site on the derivative of a pulse is also studied by the authors. This means piezoelectric sensor based mechanical technique can also be used for the recording Acceleration Plethysmogram.
\end{abstract}

Keywords: Acceleration Plethysmogram; Derivatives, Optical Pulse; Plethysmogram; Photoplethysmogram; Pressure Pulse

\section{Introduction}

Optical Signal: In the medical field, non-invasive diagnostics are mostly preferred. Because of features like simple in construction, compact, easy to use and inexpensive, these photon (sensing element) based optical sensors are becoming important. Optical sensing such as photoplethysmography is capable of measuring blood volumetric changes in the subcutaneous vessels [1]. The transmission type measurement has limitations based on measurement site. They can be used at site that has a short path length for light e.g. index finger. This limitation is overcome by the reflectance type of sensors. They can be conveniently applied on most of the body parts irrespective of the path length for light. During the work authors have acquired the PPG signal at different sites such as index finger, toes and neck using reflection type of PPG sensor. The acquired photoplethysmograph contains information about the heart rate, blood pressure, blood vessel pulsations, oxygen saturation, breathing, cardiac output and many more. The work is carried out by the authors to study the vascular ratios obtained from the optical and mechanical signal to check the suitability of pressure signal in predicting peripheral

Received (July 5, 2017), Review Result (August 7, 2017), Accepted (August 30, 2017) 
vascular disease. PPG and PP signal was utilized for obtaining the First and Second Derivatives. For arterial stiffness estimation and the evaluation of cardiovascular ageing, second derivative of PPG (SDPPG) is used. SDPPG is also known as acceleration photoplethysmogram (APG). [2,3]

Pressure Signal: When blood volume changes at peripheral site with every heartbeat is captured by using a mechanical sensor, then the captured signal is known as pressure pulse. It is the indirect estimator of blood pressure and it is a very important mortality predictor.

\subsection{Instrumentation of Optical Signal}

The PPG signal has an AC and DC component. AC component is synchronous with the heart rate and depends both on pulsatile pressure and pulsatile blood volume changes. Slow varying DC component reflects variations in total blood volume of examined tissue. The AC pulse shapes indicates vessel compliance, cardiac performance and the amplitude is usually 1-2 \% of DC value. This component is due to the tissues and average amount of blood volume that always remain in the section of the artery being sampled. The AC high signal covers the frequency region from $0.5 \mathrm{~Hz}$ to $4 \mathrm{~Hz}$. AC component is directly proportional to variation in blood volume with every heart beat caused by the pressure pulse of the cardiac cycle [4].

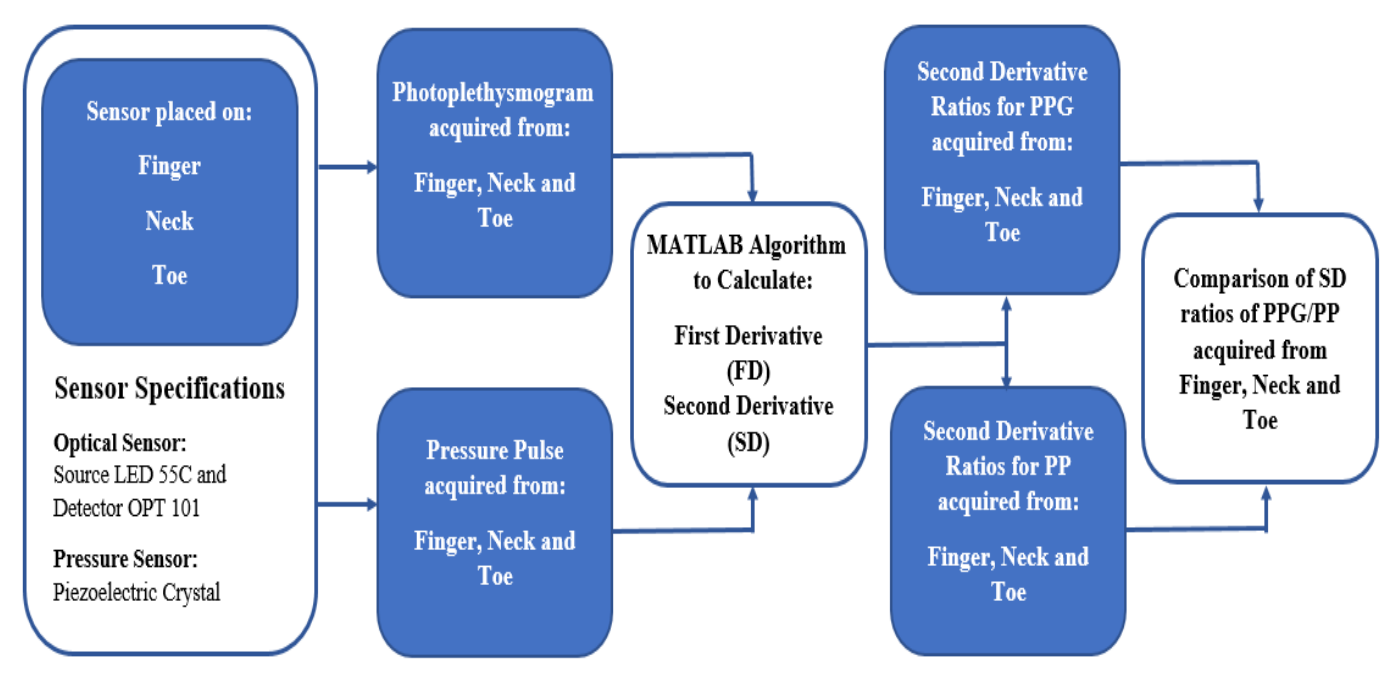

Figure 1. System Block Diagram

Figure 1 shows the block diagram representation of the work carried out.

\section{Materials and Methods}

\subsection{Optical Signal and Pressure Signal Acquisition}

Optical signal is captured using a sensor developed by the authors (as shown in Figure 2 (a)). In this sensor, IR LED - LED 55C (940 $\mathrm{nm}$ wavelength) is used as a source and OPT 101 is used as a detector. The OPT 101 is a monolithic photodiode with on-chip trans impedance amplifier. The range of the detector varies from visible to Infrared. Output voltage increases linearly with light intensity [5]. PPG can be captured by using RED LED (660 nm wavelength). For the study of derivatives of PPG, wavelength of the light source does not matter. As finally in the acceleration photoplethysmogram (second derivate of PPG) various ratios are studied to see the effect of change in PPG with respect to age/other physiological parameters. [19] 


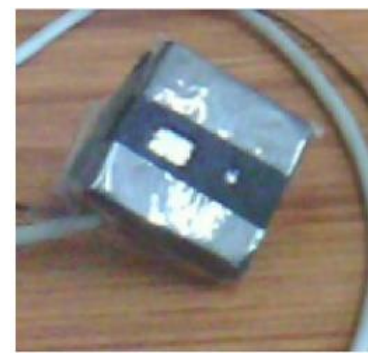

(a)

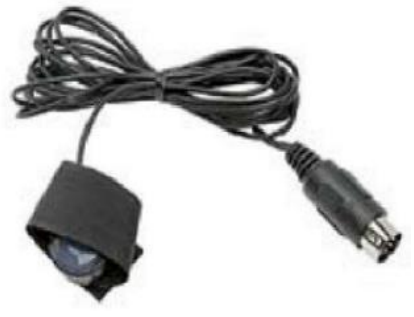

(b)

Figure 2. (a) Optical Sensor (b) Pressure Sensor [1]

ADInstruments based Power Lab hardware and software is used to acquire a pressure pulse. TN1012/ST is the piezoelectric pulse transducer (as shown in Figure 2(b)) used to record the mechanical signal (pressure pulse) from the peripheral sites [5]. Table 1 shows the comparative study of optical sensor and pressure sensor used during the study.

Table 1. Specifications of Pressure Sensor and Optical Sensor [17]

\begin{tabular}{ll}
\hline Pressure Sensor & Optical Sensor \\
\hline Piezoelectric Crystal & $\begin{array}{l}\text { Source: } 940 \mathrm{~nm} \text { (5 mm LED-55C) and } \\
\text { Detector: OPT 101 (Silicon photodiode) }\end{array}$ \\
\hline Casing: Rubber & Casing: Black Polyurethane \\
\hline Excitation: $230 \mathrm{~V}$ AC to Signal & Excitation: 12V DC-Signal Conditioning \\
Conditioning Box. & Circuit and 10V, 2 MHz AC to source \\
\hline Manufacturer: Power Lab & Manufacturer: Designed by the authors \\
\hline Weight: $~ 20$ gm & Weight: $~ 20$ gm \\
\hline Size: $1 \mathrm{~cm}$ diameter & Size: $2 \times 2.5 \mathrm{~cm}$ (size can be further \\
& reduced by keeping inner PCB smaller) \\
\hline Shape: Circular & Shape: Rectangular \\
\hline Cable: Non shielded & Cable: Shielded - 4 core \\
\hline Measurement Site: Finger, Toe and & $\begin{array}{l}\text { Measurement Site: Anywhere on the } \\
\text { human body from head to toe. }\end{array}$ \\
\hline
\end{tabular}

\subsection{First and Second Derivatives}

The first and second derivatives of the PPG \& PP were calculated to accurately recognize the critical points in both the signals. The first differentiator calculates the differences between two consecutive samples of the signal. This kind of differentiator works as a high-pass filter [6]. However, noise is higher frequencies in the PPG/PP signal. The first-order derivative parameters (as shown in Figure 3) have a non-acceptable variation and therefore are generally not used and its main features are: $[2,18]$

- $\Delta \mathrm{T}$ : It is the peak-to-peak time which is related to the time taken for the pressure wave to propagate from the heart to periphery and back. $\Delta \mathrm{T}$ is the time between the systolic and diastolic peaks in the PPG/PP signal. The definition of $\Delta \mathrm{T}$ depends on the PPG/PP waveform as its contour varies with subjects.

- Crest Time (CT): It is the time from the foot of the PPG/PP waveform to its peak. It is a useful feature for cardiovascular disease classification. 


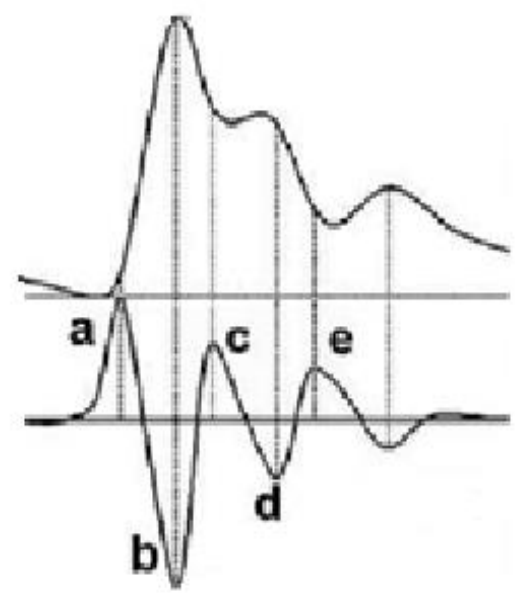

Second Derivative of Finger PPG

Figure 3. (a) Original Fingertip PPG; (b) First Derivative of Finger PPG [18]

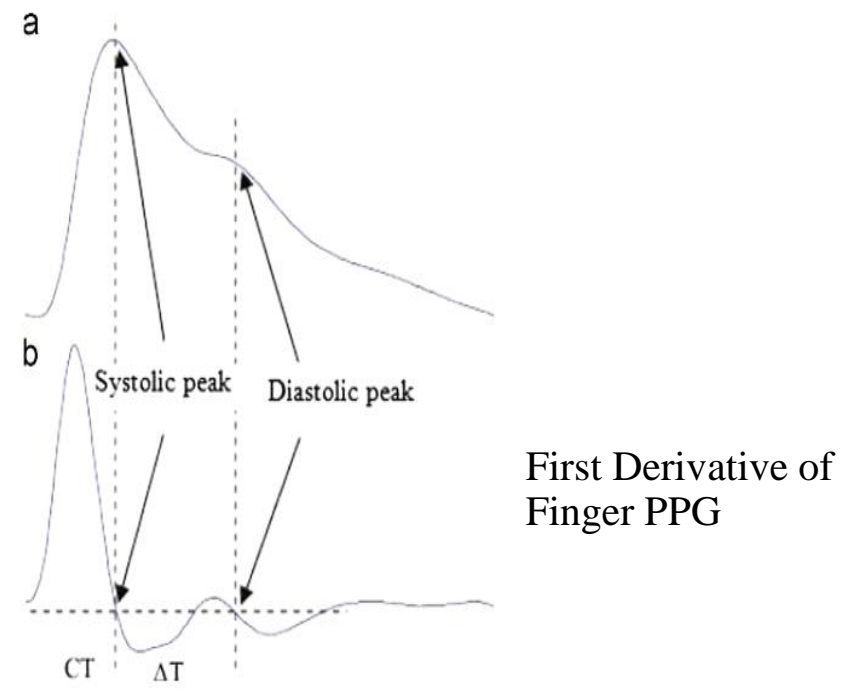

Figure 4. PPG Signal and Second Derivative of PPG with Five Distinctive High and Low Points [18]

As the first-order derivative parameters (crest time and delta $\mathrm{T}$ ) have a non-acceptable variation, the PPG/PP signal is differentiated second time to eliminate the noise and obtain a better response. The acceleration Plethysmogram (APG) mainly comprises of five distinct parts and is analyzed by using the amplitudes of the distinctive waves a, b, c, $\mathrm{d}$, and 'e', which are situated in the systolic phase of the heart cycle (as shown in figure 4). $[7,8]$

The a-e peaks in AGP: Initial positive is denoted as 'a', early negative is ' $b$ ', reincreasing point is ' $c$ ', late re-decreasing point is ' $\mathrm{d}$ ' and diastolic positive is denoted as 'e'. The relative heights of these waves (b/a, c/a, d/a and e/a ratios), are related to age, arterial blood pressure, large artery stiffness [9]. The height of each wave was measured from the baseline, the values above the baseline are positive and those under the baseline are negative. The SDPPG main ratios are: $[10,11]$

- Ratio b/a: It reflects increased arterial stiffness; hence the b/a ratio increases with age. The magnitude of b/a is related to the distensibility of the peripheral artery, it is a useful non-invasive index of atherosclerosis. b/a, is positively correlated to the Framingham Risk Score. Individual risk of cardiovascular heart disease is estimated using Framingham Risk Score. 
- Ratio c/a: It reflects decreased arterial stiffness, hence the c/a ratio decreases with age. The c/a index distinguishes subjects with essential hypertension from healthy controls.

- Ratio d/a: It reflects decreased arterial stiffness, hence the d/a ratio decreases with age. The $\mathrm{d} / \mathrm{a}$ ratio is useful for the evaluation of vasoactive agents, and is an index of left ventricular afterload.

- Ratio e/a: An increase of the e/a ratio reflects decreased arterial stiffness, and that the e/a ratio decreases with age.

- Ratio (b-c-d-e)/a (Aging Index): The aging index (AGI) is used to describe the cardiovascular age of the subject. The (b-c-d-e)/a index, increases with age and is useful for evaluation of vascular aging and for screening of arteriosclerotic disease. $[7,12,18]$

\subsection{Statistical Analysis}

Analysis of acceleration PPG and acceleration PP is carried out by studying three statistical features; such as mean, standard deviation and Pearson product.

- Mean: Addition of all the data points divided by the total number of data points gives the mean value for that data set. It is the average value of the data set.

- Standard Deviation: It is one of the very important and commonly studied statistical feature. It is the measure of dispersion or variation from the average or mean value of the data set. High standard deviation shows that the data points are widely spread out, while lower standard deviation shows that the data points are less dispersed. $[4,13]$

- Pearson Product: Pearson Product returns the moment correlation coefficient ' $r$ ', which ranges from -1 to 1 . It is one of the normalized measures of coupling. +1 indicate positive correlation ( $\mathrm{X}$ increases as $\mathrm{Y}$ increases), zero indicate no correlation and -1 indicate negative correlation ( $\mathrm{Y}$ decreases as $\mathrm{X}$ increases).

\section{Results}

PPG and PP was captured at various sites on the human body such as finger, neck and toe. First and second derivative related parameters for all the signals were calculated. Figure 5 shows the pressure pulse captured at finger, neck and toe and its first and second derivative. Velocity Plethysmogram (first derivative of PPG and PP) related Crest Time $(\mathrm{CT})$ and Delta $\mathrm{T}(\Delta \mathrm{T})$ are the two parameters studies by the authors. It was observed that the mean value and standard deviation of $\mathrm{CT}$ and $\Delta \mathrm{T}$ for PPG are: $48.90566 \pm 5.299725 \&$ $78.4528 \pm 10.3637$; for PP are: $39.116279 \pm 3.2012594 \& 69.76744 \pm 11.245$ respectively. 


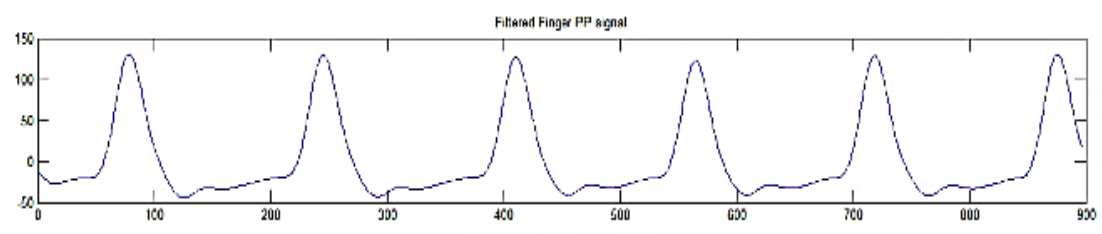

(a) Finger PP

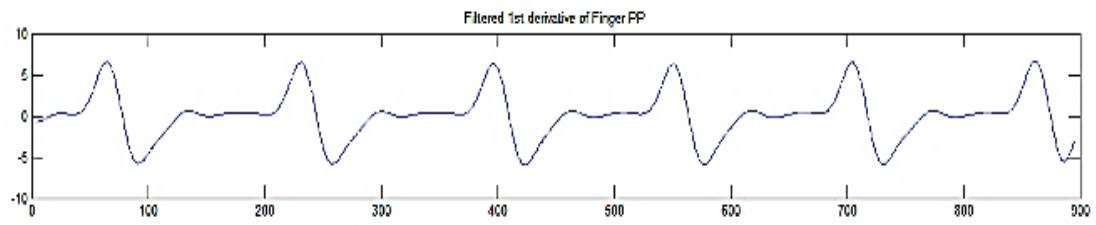

(b) First Derivative of Finger PP

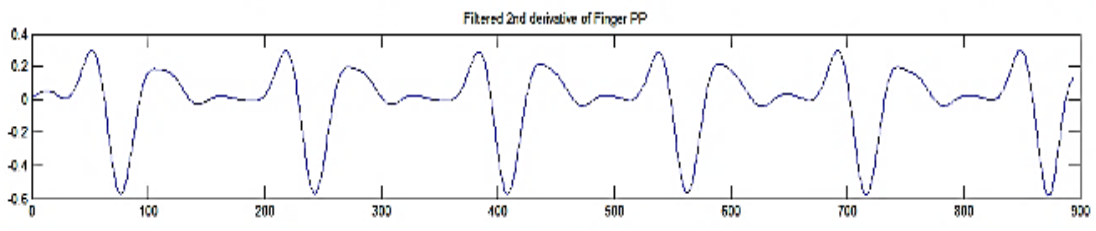

(c) Second

Derivative of Finger PP

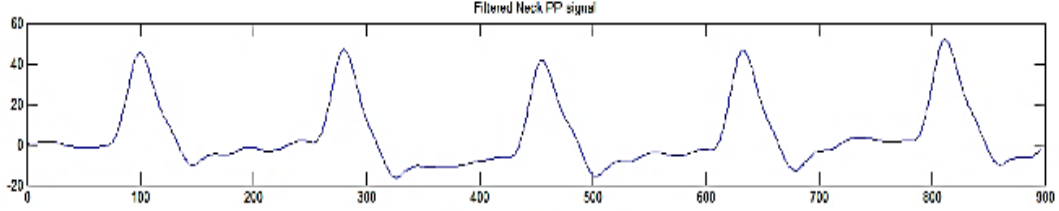

(d) Neck PP

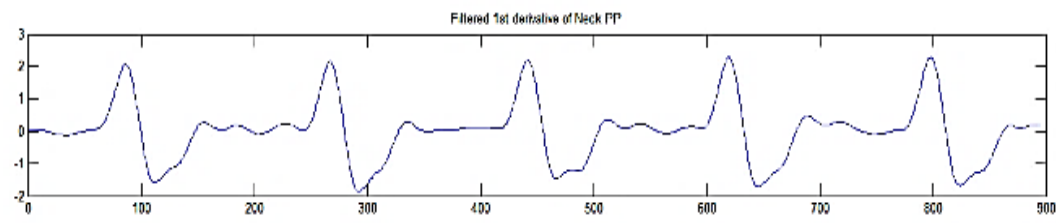

(e) First Derivative of Neck PP

Fitersos 2rod detirestiv of Neck $F$

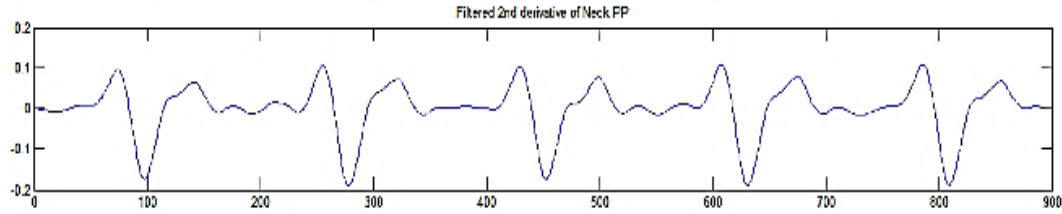

(f) Second

Derivative of Neck PP

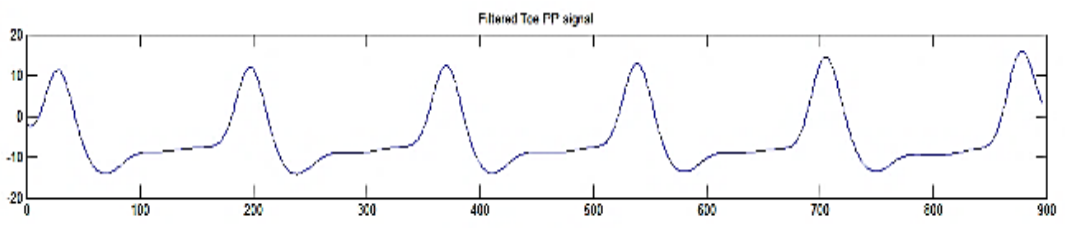

(g) Toe PP

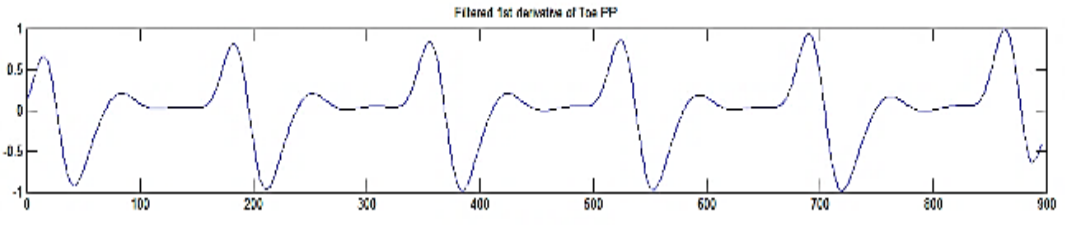

(h) First Derivative of Toe PP

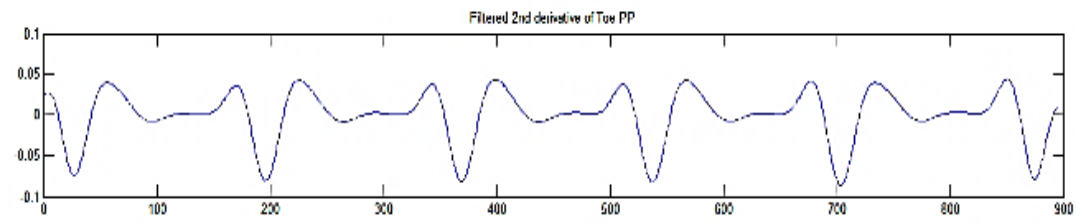

(i) Second

Derivative of Toe PP

Figure 5. (a-b-c) Pressure Pulse at Finger and its First and Second Derivative, (d-e-f) Pressure Pulse at Neck and its First and Second Derivative $(\mathrm{g}-\mathrm{h}-\mathrm{i})$ Pressure Pulse at Toe and its First and Second Derivative 
Table 2 shows all the second derivative related ratios and its mean value along with the standard deviation for PPG \& PP are as follows. Table 3 shows Pearson product of second derivative related parameters of optical pulse (PPG) \& pressure pulse (PP) as follows.

Table 4 shows the $\mathrm{P}$-value of the t-paired test carried out on the means of various (b/a, c/a. d/a, e/a and (b-c-d-e)/a) ratios studied from acceleration (Second Derivative) photoplethysmogram/pressure pulse acquired from finger, neck and toe. Results of $t$ paired test calculated for alpha equal to 0.05 .

Table 5 shows the P-value of the t-paired test carried out on the means of five ratios obtained from second derivative of photoplethysmogram at Finger - Neck, Finger - Toe and Neck - Toe. Similarly, to find out P-value the t-paired test is carried out on five ratios obtained from second derivative of Pressure Pulse at Finger - Neck, Finger - Toe and Neck - Toe. EXCEL 2016 software is used to calculate the P-value. It provides the clear idea about the mean values of second derivative ratios obtained from PPG or PP at different measurement sites.

Table 2. Mean \& Standard Deviation of Acceleration Photoplethysmogram \& Acceleration Pressure Plethysmogram Related Ratios

\begin{tabular}{lll}
\hline & \multicolumn{2}{c}{ Measurement Site: Finger } \\
\hline Ratio & $\begin{array}{l}\text { PPG: Mean } \pm \text { Standard } \\
\text { Deviation }\end{array}$ & PP: Mean \pm Standard Deviation \\
b/a & $-0.51619 \pm 0.16320$ & $-1.73914 \pm 0.14700$ \\
c/a & $0.26383 \pm 0.11368$ & $0.66657 \pm 0.13111$ \\
d/a & $-0.20829 \pm 0.10757$ & $-0.25997 \pm 0.25453$ \\
e/a & $0.14332 \pm 0.07280$ & $0.14965 \pm 0.10491$ \\
(b-c-d-e)/a & $-0.70711 \pm 0.29825$ & $-2.29540 \pm 0.31257$ \\
\hline \multicolumn{3}{c}{ Measurement Site: Neck } \\
\hline Ratio & PPG: Mean \pm Standard Deviation & PP: Mean \pm Standard Deviation \\
b/a & $-0.54890 \pm 0.14400$ & $-1.63307 \pm 0.40901$ \\
c/a & $0.29193 \pm 0.10969$ & $0.57967 \pm 0.21761$ \\
d/a & $-0.29388 \pm 0.09592$ & $-0.21755 \pm 0.13547$ \\
e/a & $0.15573 \pm 0.07080$ & $0.19844 \pm 0.10183$ \\
(b-c-d-e)/a & $-0.70269 \pm 0.18405$ & $-2.19364 \pm 0.54798$ \\
\hline \multicolumn{2}{c}{ Measurement Site: Toe } \\
\hline Ratio & PPG: Mean \pm Standard Deviation & PP: Mean \pm Standard Deviation \\
b/a & $-0.64267 \pm 0.15103$ & $-1.72111 \pm 0.21292$ \\
c/a & $0.29937 \pm 0.10846$ & $0.75631 \pm 0.12124$ \\
d/a & $-0.22064 \pm 0.10136$ & $-0.27648 \pm 0.14590$ \\
e/a & $0.13325 \pm 0.04329$ & $0.11006 \pm 0.09224$ \\
(b-c-d-e)/a & $-0.85466 \pm 0.20784$ & $-2.31100 \pm 0.29969$ \\
\hline
\end{tabular}


Table 3. Pearson Product of Acceleration Photoplethysmogram and Acceleration Pressure Plethysmogram Related Features

\begin{tabular}{lll}
\hline & \multicolumn{2}{c}{ Measurement Site: Finger } \\
\hline Parameter & PPG: Pearson Product & PP: Pearson Product \\
a \& b & -0.96861 & -0.98857 \\
a \& c & 0.98380 & 0.94506 \\
a \& d & -0.84870 & -0.33590 \\
a \& e & 0.97105 & 0.50808 \\
\hline \multicolumn{3}{c}{ Measurement Site: Neck } \\
\hline Parameter & PPG: Pearson Product & PP: Pearson Product \\
a \& b & -0.95067 & -0.98280 \\
a \& c & 0.96118 & 0.21169 \\
a \& d & -0.94990 & -0.66738 \\
a \& e & 0.96002 & 0.74005 \\
\hline & \multicolumn{2}{c}{ Measurement Site: Toe } \\
\hline Parameter & PPG: Pearson Product & PP: Pearson Product \\
a \& b & -0.85454 & -0.98103 \\
a \& c & 0.76631 & 0.98366 \\
a \& d & -0.46988 & -0.94994 \\
a \& e & 0.73545 & 0.44855 \\
\hline
\end{tabular}

Table 4. $P$ Value for Means of Ratios of Second Derivative Obtained from PPG and PP

\begin{tabular}{|c|c|}
\hline \multicolumn{2}{|c|}{$\begin{array}{c}\text { PPG/PP - Measurement Site: Finger, Neck and } \\
\text { Toe }\end{array}$} \\
\hline Ratio & $P$ value \\
\hline $\mathrm{b} / \mathrm{a}$ & 0.000874 \\
\hline $\mathrm{c} / \mathrm{a}$ & 0.008294 \\
\hline $\mathrm{d} / \mathrm{a}$ & 0.416456 \\
\hline e/a & 0.347740 \\
\hline$(b-c-d-e) / a$ & 0.000340 \\
\hline
\end{tabular}

Table 5. $P$ Value for Means of all the Five Ratios of Second Derivative Obtained from Photoplethysmogram and Pressure Pulse from Various Measurement Sites

\begin{tabular}{ll}
\hline \multicolumn{2}{c}{ For Photoplethysmogram } \\
\hline Measurement Site & P value \\
Finger - Neck & 0.255372 \\
Finger - Toe & 0.109483 \\
Neck - Toe & 0.196575 \\
\hline \multicolumn{2}{c}{ For Pressure Pulse } \\
\hline Measurement Site & P value \\
Finger - Neck & 0.145369 \\
Finger - Toe & 0.382697 \\
Neck - Toe & 0.274116 \\
\hline
\end{tabular}

\section{Discussion}


PPG wave being flat has a limitation on analysis of wave changes. To make these weak changes prominent, differentiated value of the original PPG wave is used. This first derivative of the PPG is also known as 'Velocity Photoplethysmogram' (VPG). This first derivative value is useful to understand process of pulse wave change, which was difficult in original PPG signal. VPG is differentiated again to more specifically realize process of pulse energy. This second derivative value is known as 'Accelerated Photoplethysmogram' (APG). PPG signal is widely used to for the cardiac parameter predictions such as 'Heart Rate', 'Blood Pressure', 'Respiratory Rate', 'Blood Oxygen Saturation' and the 'Cardiac Output'. Widely studied first derivative of PPG based predictions are 'Crest Time' and 'Delta T'. Second derivative of PPG based common predictions are various ratios such as b/a, c/a, d/a, e/a and (b-c-d-e)/a ratio. APG related standard values of various ratios for the heathy subject are approximately; b/a: -0.9 $(\mathrm{SD}=0.09)$, c/a: $0.2(\mathrm{SD}=0.09), \mathrm{d} / \mathrm{a}:-0.1(\mathrm{SD}=0.1)$ and e/a: $0.1(\mathrm{SD}=0.1)$ [14]. Change in vascular elasticity can be found out from second derivative of PPG [3]. Sometimes fourth order derivative of PPG is calculated to study the changes in the vascular systems health [15]. PPG derivatives are also used as one of the important biometric identifier. $[4,6,16]$

\section{Limitation}

Optical signal and pressure signal are the only two signals studied by the authors in this paper. These signals were captured from normal subjects without any known heart related problem or blood glucose related problem. Vascular health mainly gets affected in heart related diseases or in blood glucose related diseases. As changes in the arterial health is prominent in subjects with cardiovascular disease or with diabetes (abnormal blood glucose levels). Same study can be carried out on subjects with either having only cardiovascular disease or diabetes. Such a study will be able to reveal how vascular health gets affected in subjects having known heart problem. Similarly, second derivative related study of PPG/PP can be carried out on subjects having only diabetes (no heart problem) to find out how vascular health gets affected on diabetes. Or study can be carried out on the subjects who are having both (cardiovascular and diabetes) diseases and then effect of these disease on vascular health can be found out along with age of the subject.

\section{Conclusion}

Aging is the process which affects every organ of the human body; vascular system is one of them. Over the years gradually arteries lose the elasticity and become hard. This process is known as arteriosclerosis. Lot of factors affect shape of the pulse wave. Sometimes arteriosclerosis and the shape changes occurs due to some diseases (such as diabetes, hypertension, hyperlipidaemia, obesity and chain smoking) without any association with the age. This affects the peripheral circulation. This change in the peripheral circulation using various ratios is studies by the authors by acquiring optical pulse (PPG) and pressure pulse (PP) at different body sites. Total 60 real signals (30 PPG signals and 30 PP signals) at finger, neck and toe for normal subjects aged between $20-$ 40 years were studied. It is observed that the APG related ratios obtained for PPG and PP captured from finger, neck and toe are very much same and are close to the standard values for the normal subjects. Measurement site has almost no effect on the features extracted from first and second derivative. This shows that the pressure pulse can also be used in future, instead of optical pulse to carry out the study related to the vascular aging. Strong negative correlation was observed between ' $a$ ' and ' $b$ ', while strong positive correlation was observed between 'a-c' and 'a-e'. Acquisition of PPG or PP from any site (not directly from heart) and its analysis can reveal accurate information related to cardiovascular system; such as BP, HR, RR, CT, $\Delta \mathrm{T}$ and APG related various ratios [17, 18]. P-value (with $\alpha=0.05$ ) of t-paired test carried out on means of all the five ratios (b/a, c/a, d/a, e/a and (b-c-d-e)/a) is shown in Table 4. From this P-value we can make out that 
irrespective of the signal acquisition site strong similarity was found between $\mathrm{d} / \mathrm{a}$ and e/a ratio $(\mathrm{P}>0.05$ ). Table 5 shows the $\mathrm{P}$-value (with $\alpha=0.05$ ) calculated based on means of all the five second derivative ratios for PPG captured from Finger - Neck $(P>0.05)$, Finger Toe $(\mathrm{P}>0.05)$ and Neck - Toe $(\mathrm{P}>0.05)$. This table also covers the $\mathrm{P}$-value (with $\alpha=0.05)$ calculated based on means of all the five second derivative ratios for PP captured from Finger - Neck $(\mathrm{P}>0.05)$, Finger - Toe $(\mathrm{P}>0.05)$ and Neck - Toe $(\mathrm{P}>0.05)$. This shows that mean value of all the five SD ratios are very similar irrespective of the signal acquisition site for both the signals (either PPG or PP). Thus, PPG or PP captured from any measurement site can be used to carry out the derivative related study in future. So optical signals or a mechanical signal can be used for the indirect prediction of the health of cardiovascular system.

\section{References}

[1] Revati Shriram, M. Sundhararajan \& Nivedita Daimiwal, "Application of High \& Low Brightness LEDs to Human Tissue to capture Photoplethysmogram at a Finger Tip", International Conference for Convergence of Technology (I2CT), $6^{\text {th }}-8^{\text {th }}$ April (2015), Pune, India.

[2] A. Re, Kavsao lu, Kemal Polat, M. Recep Bozkurt, "A novel feature ranking algorithm for biometric recognition with PPG signals", Computers in Biology \& Medicine 49 (2014) pp 1 -14.

[3] Jianchu Yao, Xiaodong Sun \& Yongbo Wan, "A Pilot Study on Using Derivatives of Photoplethysmographic Signals as a Biometric Identifier", 29th Annual International Conference of the IEEE EMBS Cité Internationale, Lyon, France, August 23-26, (2007).

[4] Dae-Geun Jang, Jang-Ho Park, Seung-Hun Park, Minsoo Hahn, "A Morphological Approach to Calculation of the Second Derivative of Photoplethysmography", $10^{\text {th }}$ International Conference on Signal Processing (ICSP), $24^{\text {th }}-28^{\text {th }}$ October (2010), Beijing, China.

[5] Revati Shriram \& M. Shundhararajan, "Coherence Analysis of Pressure Pulse and Photoplethysmogram at various sites", International Journal of Applied Engineering Research (IJAER), Vol 10, No 6, PP 14959-14968, (2015).

[6] Nurhafizah Mahri, Kok Beng Gan, and Mohd. Alauddin Mohd. Ali, "Extracting Features Similar to QT Interval from Second Derivatives of Photoplethysmography: A Feasibility Study", 2014 IEEE Conference on Biomedical Engineering and Sciences, 8 - 10 December (2014), Miri, Sarawak, Malaysia.

[7] Q. Yousef, M. B. I. Reaz, M. A. M. Ali, "The Analysis of PPG Morphology: Investigating the Effects of Aging on Arterial Compliance", Measurement Science Review, Volume 12, No. 6, (2012).

[8] R. Mohamad Rozi, M. A. Mohd Ali, M. B. I. Reaz, "Effects of Exercise on the Second Derivative Photoplethysmography (PPG) Waveform", Asia Pacific Conference on Circuits and Systems (APCCS), $6^{\text {th }}-9^{\text {th }}$ December (2010), Kuala Lumpur, Malaysia.

[9] Yousef K. Qawqzeh, Rubins Uldis and Mafawez Alharbi, "Photoplethysmogram second derivative review: Analysis and applications", Academic Journal, Scientific Research and Essays, Vol. 10(21), pp. 633-639, 15 November, (2015) DOI: 10.5897/SRE2015.6322 Article Number: A214BA956534 ISSN 1992-2248.

[10] Sahnius bt Usman, Mohd Alauddin bin Mohd Ali, Md. Mamun Bin Ibne Reaz, Kalaivani Chellapan, "Second Derivative of Photoplethysmogram in Estimating Vascular Aging among Diabetic Patients" International Conference on Technical Postgraduates (TECHPOS), $14^{\text {th }}-15^{\text {th }}$ December (2009), Kuala Lumpur, Malaysia.

[11] Dae-Geun Jang, Jang-Ho Park, Seung-Hun Park, Minsoo Hahn, "A Morphological Approach to Calculation of the Second Derivative of Photoplethysmography", $10^{\text {th }}$ International Conference on Signal Processing (ICSP), $24^{\text {th }}-28^{\text {th }}$ October (2010), Beijing, China.

[12] R. Mohamad Rozi, Sahnius Usman, M.A. Mohd Ali,M.B.I Reaz, "Second Derivatives of Photoplethysmography (PPG) for Estimating Vascular Aging of Atherosclerotic Patients", 2012 International Conference on Biomedical Engineering and Sciences | Langkawi | 17th - 19th December (2012).

[13] Revati Shriram, M. Sundhararajan, Sukhada Unde \& Nivedita Daimiwal, "Statistical Analysis of Connectivity Measures of Electroencephalogram during Colored Word Reading Interference", International Conference on Emerging Novelties \& Vistas in Space Technologies \& Applications" (ENVISTA), $25^{\text {th }}-27^{\text {th }}$ February (2016), Chennai, India.

[14] I. Jeong, J. Finkelstein, "Applicability of the Second Derivative Photoplethysmogram for Non-invasive Blood Pressure Estimation during Exercise”, (2013) Pan American Health Care Exchanges (PAHCE).

[15] Jae Mok Ahn, "Wave Detection in Acceleration Plethysmogram", Health Inform Res. (2015) April;21(2):111-117, pISSN 2093-3681 • eISSN 2093-369X.

[16] Nur Azua Liyana Jaafar, Khairul Azami Sidek and Siti Nurfarah Ain Mohd Azam, "Acceleration Plethysmogram Based Biometric Identification", (2015) International Conference on BioSignal 
Analysis, Processing and Systems (ICBAPS), Kuala Lumpur, Malaysia.

[17] Revati Shriram, M. Sundhararajan \& Nivedita Daimiwal (2015), "Statistical Analysis of Pulse Acquired from various Body sites using Piezoelectric and Optical Transducer”, XII Control Instrumentation System Conference (CISCON), $2^{\text {nd }}-4^{\text {th }}$ November (2015), Manipal, India.

[18] Elgendi M, Norton I, Brearley M, Abbott D, Schuurmans D (2013) Systolic Peak Detection in Acceleration Photoplethysmograms Measured from Emergency Responders in Tropical Conditions. PLoS ONE 8(10): e76585. doi:10.1371/journal.pone.0076585.

[19] Revati Shriram, Betty Martin, M. Sundhararajan and Nivedita Daimiwal (2016), "Effect of Source Wavelength on Second Derivative of Finger Photoplethysmogram in Healthy Young Female Volunteers", Biomedical Research, ISSN: 0970-938X.

\section{Authors}

Revati Shriram, she received her Bachelor's Degree in Instrumentation and Control from Cummins College of Engineering for Women, Pune, India. Received her Master's Degree in Electrical Engineering from Rose-Hulman Institute of Technology, Indiana, USA. Currently she is working as an Assistant Professor in the department of Instrumentation and Control of Cummins College of Engineering for Women. She is pursuing her Doctorate from Sathyabama University, Chennai, India.

Betty Martin, she is a Professor in the department of Electronics and Control of Sathyabama University Chennai, India.

M Sundhararajan, he is a Professor and Dean Research at Bharath University, Chennai, India.

Nivedita Daimiwal, she is a Assistant Professor in the department of Instrumentation and Control of Cummins College of Engineering for Women, Pune, India. 
International Journal of Signal Processing, Image Processing and Pattern Recognition Vol. 10, No. 9 (2017) 